\title{
Premières données sur la présence de l'abeille asiatique Megachile (Callomegachile) sculpturalis SMITH (Hymenoptera, Megachilidae) en Europe
}

\author{
Par Nicolas J VereeCKeN et Eric BARBIER ${ }^{\star *}$
}

\begin{abstract}
Abeilles «baladeuses»
La plupart des abeilles solitaires sont généralement peu mobiles une fois installées dans un environnement propice : on estime que la distance entre le site de nidification et les ressources florales exploitées pour le pollen indispensable au développement de la progéniture varie de quelques mètres à quelques centaines de mètres la plupart du temps (Kapyla 1978; Eickwort \& Ginsberg 1980; Gathmann \& Tscharnke 2002; Franzén et al. 2009; voir aussi Greenleaf et al. 2007). Les possibilités de dispersion entre populations distantes de plusieurs dizaines, voire plusieurs centaines de kilomètres semblent donc relativement limitées $a$ priori.
\end{abstract}

La famille des Megachilidae comprend de nombreuses espèces qui nidifient dans des cavités creusées dans le bois mort ou dans des tiges par elles-mêmes ou par d'autres insectes (Michener 2007). Ce mode de nidification a par exemple permis à certains groupes d'espèces comme les Hoplitis, les Protosmia et les Osmia (Helicosmia) nidifiant dans le bois de conquérir le Nouveau Monde par voie maritime, alors que les espèces terricoles dont elles sont dérivées sont limitées à l'Ancien Monde (Michener 1979, 2007; Griswold 1986; Cane et al. 2007; Praz et al. 2008; voir aussi Schwarz et al. 2006). L'intensification du commerce du bois et de son transport le long des grands axes maritimes font des régions portuaires des lieux-clés d'arrivée d'espèces d'insectes xylicoles et de leurs nids (Boersma et al. 2006). A l'échelle européenne, la récente découverte de Xylocopa violacea (Hymenoptera, Apidae) nidifiant dans de vieux pommiers dans le secteur de Shepshed en Angleterre (Peat 2007) indique que cette introduction s'est vraisemblablement faite depuis la plate-forme de fret ferroviaire de Loughborough (Leicester) où arrivent les stocks de bois en provenance d'Europe continentale et d'ailleurs. Le même type de phénomène est probablement responsable de l'installation de populations de $X$. (Neoxylocopa) darwinii, la seule espèce d'abeille sauvage indigène des îles Galapagos (Linsley et al. 1966; McMullen 1993; Philipp et al. 2006).

\footnotetext{
* Evolution Biologique et Ecologie, Université Libre de Bruxelles CP 160/12, Av. F.D. Roosevelt 50, B-1050 Bruxelles, Belgique. E-mail: nicolas.vereecken@ulb.ac.be

** Avenue du Général Leclerc 356, F-13190 Allauch, France E-mail: fiem13@free.fr
}

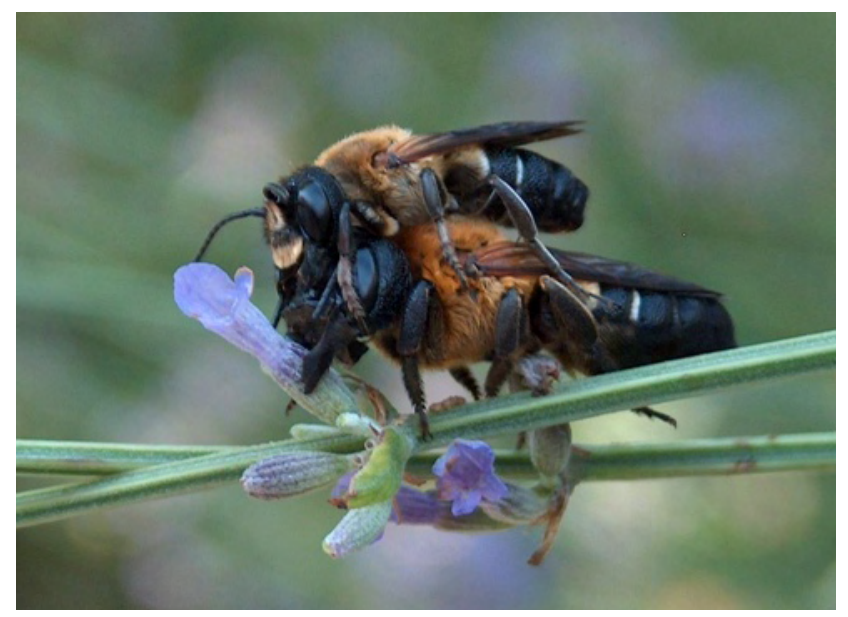

Figure 1. Couple de Megachile sculpturalis observé sur une tige de lavande, 2.VII.2008, Allauch (France) (Photo E Barbier)

\section{Une abeille asiatique en Europe}

Début juillet 2008, l'un d'entre nous (EB) a réalisé une série de macrophotographies d'une abeille solitaire dans les environs d'Allauch, à proximité du port de Marseille (France) (Figures 1 et 2). L'examen des photographies a rapidement indiqué que l'insecte observé n'était en aucun cas une espèce européenne, mais qu'il s'agissait sans aucun doute de Megachile (Callomegachile) sculpturalis SMITH. Cette espèce de grande taille peut atteindre $20 \mathrm{~mm}$ et est originaire d'Asie orientale (Chine, Corée, Japon et Taiwan) (Iwata 1933; Wu 2005) et a été découverte pour la première fois en Caroline du Nord (USA) en 1994 (Mangum \& Brooks 1997; Batra 1998) d'où elle s'est répandue pour atteindre le Kansas à l'Ouest (Hinojosa-Diaz 2008) et Ontario (Canada) au Nord depuis 2002 (Mangum \& Somner 2003). L'espèce semble même être devenue particulièrement abondante dans la région de $\mathrm{New}$ York (Ascher 2001; Matteson et al. 2008; BN Danforth, pers. comm. 2009).

Les femelles de $M$. sculpturalis construisent les cellules larvaires au sein de leur nid à l'aide de résine, les séparant les unes des autres à l'aide de terre, le plus souvent au sein de cavités pré-existantes dans des tiges de bois ou dans du bois mort (Tsuneki 1970). Cette espèce appartient au sous-genre (sub)tropical Callomegachile qui compte parmi ses représentants une autre espèce spectaculaire, $M$. pluto, l'une des plus grandes abeilles solitaires au monde (Figure 3). Les 

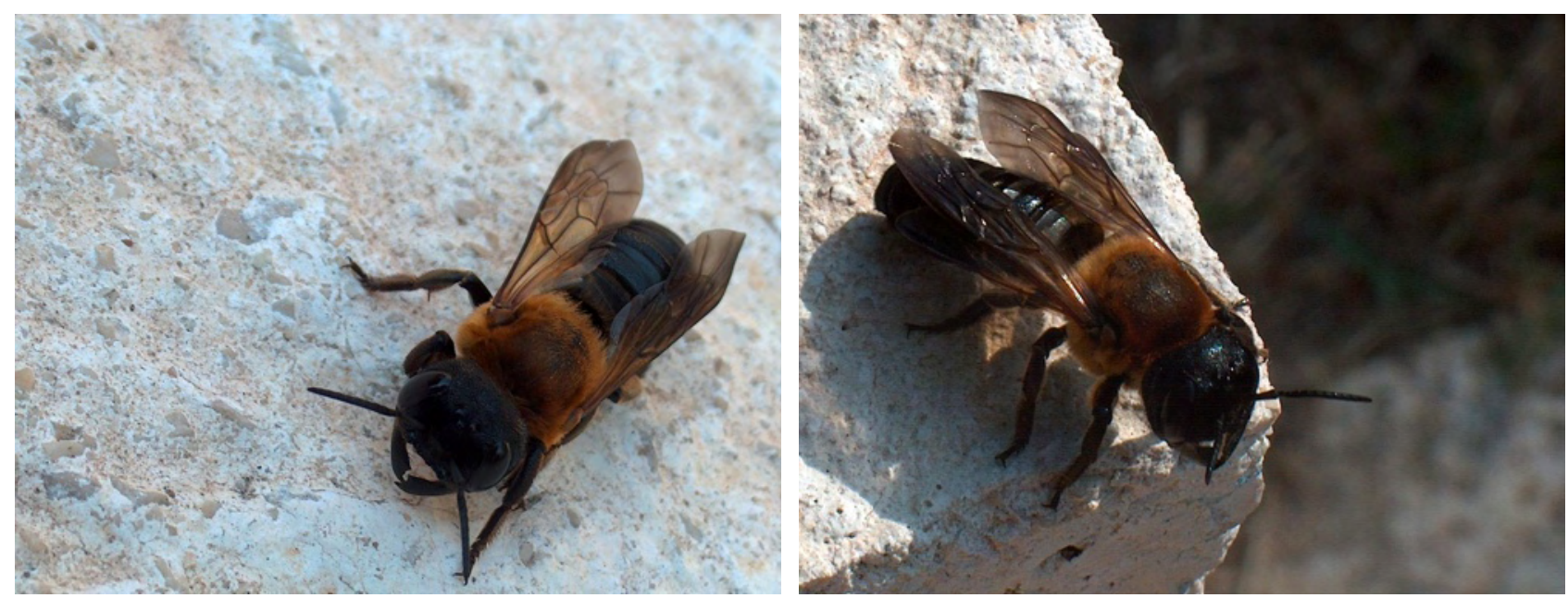

Figure 2. Femelle de Megachile sculpturalis (Hymenoptera, Megachilidae), 2.VII.2008, Allauch (France) (Photo E Barbier)

femelles de $M$. pluto nidifient dans les termitières et utilisent leurs extraordinaires mandibules pour récolter de la résine et des fibres végétales qui serviront de base à l'élaboration des cellules larvaires (Messer 1984). La biologie et la taille de $M$. sculpturalis lui ont valu le surnom de «giant resin bee» aux Etats-Unis où un programme de suivi de son expansion géographique est en place depuis la fin des années 1990. L'espèce semble être largement polylectique (Mangum \& Sumner 2003).

\section{Discussion}

De nombreuses espèces d'abeilles du genre Megachile ont déjà été trouvées sur les îles océaniques et en dehors de leur aire de distribution (Raw 2004). L'implantation de ces espèces exotiques répond aux mêmes exigences que celles qui s'appliquent à nos abeilles locales : nécessité d'un site de nidification adéquat, ressources florales disponibles, et conditions climatiques ad hoc.

La découverte de plusieurs spécimens $(\mathrm{M} / \mathrm{F})$ de $M$. sculpturalis dans le sud de la France, non loin de la zone portuaire de Marseille illustre parfaitement les capacités de dispersion de certaines espèces d'abeilles solitaires et ce parfois sur des distances d'ordre intercontinental. Le caractère polylectique de cette espèce lui permettront peut-être d'outre-passer les contraintes alimentaires liées à la colonisation des habitats du sud de la France, et il restera à déterminer si l'espèce est susceptible de s'installer durablement dans nos régions voire de s'y répandre à la manière de ce qui est observé actuellement aux Etats-Unis (voir références citées cidessus).

\section{Remerciements}

Nous remercions Gérard LeGoff (Barentin, France) et Christophe Praz (ETH Zürich, Suisse) qui ont eu la gentillesse de se pencher sur les photos illustrant cet article, permettant ainsi l'identification des spécimens observés, ainsi que Bryan Danforth pour ses commentaires sur la présence de Megachile sculpturalis dans l'Etat de New-York. La photographie de la Figure 3 a été réalisée grâce à l'aide de Darren Mann du Musée d'Oxford.

\section{Références bibliographiques}
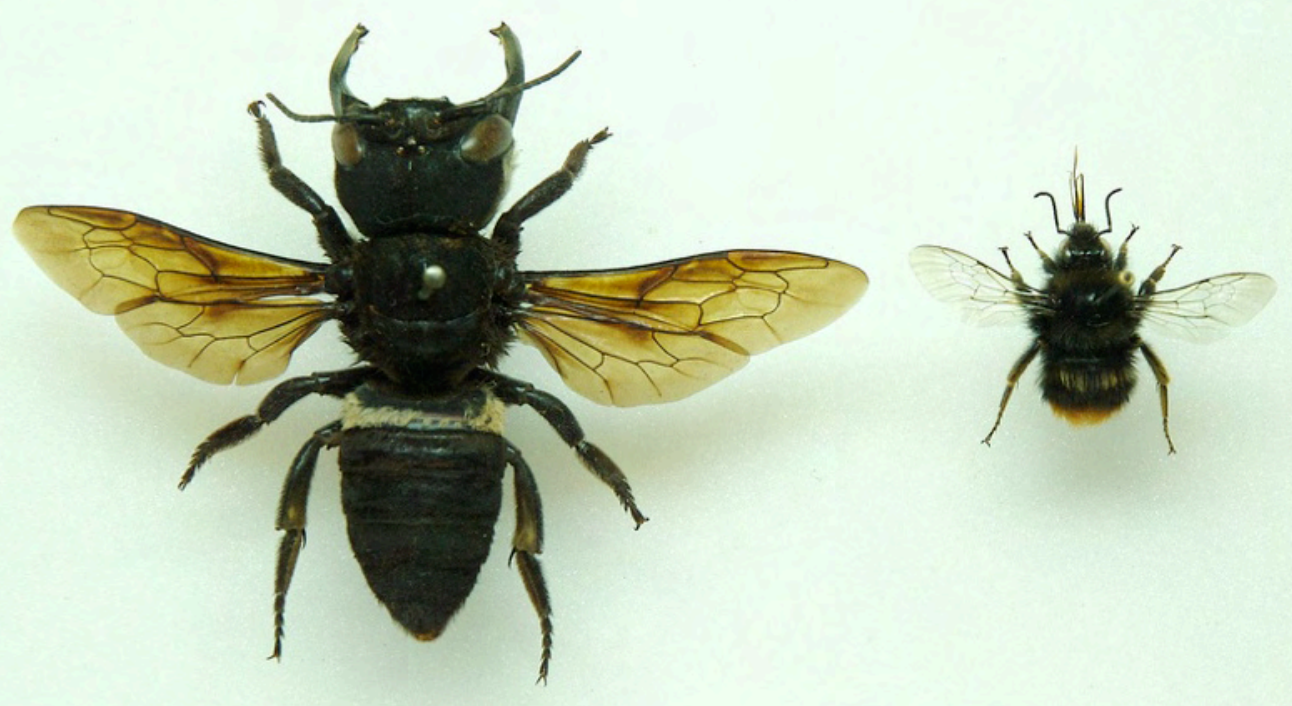

Figure 3. Megachile (Callomegachile) pluto SMITH (Hymenoptera, Megachilidae) (gauche), mâle de Bombus ruderarius (MüLLER) (Hymenoptera, Apidae) (centre) et femelle de Ceratina parvula SMITH (Hymenoptera, Apidae). Le spécimen de M. pluto photographié est le type récolté par Alfred Russel WALLACE en Indonésie et conservé dans la collection entomologique du Musée de I'Université d'Oxford (Angleterre) (Photo NJ Vereecken). 


\section{OSMIA n³ - 2009}

Ascher JS, 2001. Hylaeus hyalinatus SMITH, a European bee new to North America, with notes on other adventive bees (Hymenoptera: Apoidea). Proceedings of the Entomological Society of Washington 103 : 184-190.

Batra SWT, 1998. Biology of the giant resin bee, Megachile sculpturalis SMITH, a conspicuous new inmigrant in Maryland. The Maryland Naturalist 42(1-2) : 1-3.

Boersma PD, Reichard SH \& Van Buren AN, 2006 (éds). Invasive species in the Pacific Northwest. University of Washington Press, USA

Cane JH, Griswold TL \& Parker FD, 2007. Substrates and materials used for nesting by North American Osmia bees (Hymenoptera: Apiformes: Megachilidae). Annals of the Entomological Society of America $100: 350-358$.

Eickwort GG \& Ginsberg HS, 1980. Foraging and mating behaviour in Apoidea. Annual Review of Entomology 25: 421-446.

Franzén M, Larsson M \& Nilsson SG, 2009. Small local population sizes and high habitat patch fidelity in a specialised solitary bee. Fournal of Insect Conservation $13: 89-95$.

Gathmann A \& Tscharntke T, 2002. Foraging ranges of solitary bees. Journal of Animal Ecology 71 : 757-764.

Greenleaf SS, Williams NM, Winfree R \& Kremen C, 2007. Bee foraging ranges and their relationship to body size. Oecologia $153: 589-596$.

Griswold TL, 1986. Notes on the nesting biology of Protosmia (Chelostomopsis) rubifloris (COCKERELL). The Pan-Pacific Entomologist $62: 84-87$

Hinojosa-Diaz IA, 2008. The giant resin bee making its way west: first record in Kansas (Hymenoptera: Megachilidae). ZooKeys 1 : 67-71.

Iwata K, 1933. Studies on the nesting habits and parasites of Megachile sculpturalis SMITH (Hymenoptera, Megachilidae). Mushi 6 : 4-26.

Kapyla M, 1978. Foraging distance of small solitary bee, Chelostoma maxillosum (Hym., Megachilidae). Annales Entomologici Fennici 44 : 63-64.

Linsley EG, Rick GM \& Stephens SG, 1966. Observation on the floral relationships of the Galápagos carpenter bee. The Pan-Pacific Entomologist 42: 1-18. the continental United States. Fournal of the Kansas Entomological Society 70(2): 140-142.

Mangum WA \& Sumner S, 2003. A survey of the North American range of Megachile (Callomegachile) sculpturalis, an adventive species in North America. Fournal of the Kansas Entomological Society 76(4) : 658-662.

Matteson KC, Ascher JS \& Langellotto GA, 2008. Bee richness and abundance in New-York city urban gardens. Annals of the Entomological Society of America 101(1) : 140-150.

McMullen CK, 1993. Flowering-visiting insects of the Galápagos Islands. The Pan-Pacific Entomologist 69(1) : 95-106.

Messer AC, 1984. Chalicodoma pluto: The world's largest bee rediscovered living communally in termite nests. Fournal of the Kansas Entomological Society 57 : 165-168.

Michener CD, 2007. The Bees of the World, second edition. The Johns Hopkins University Press, Baltimore.

Peat L, 2007. Xylocopa violacea excitement. BWARS Neresletter, Autumn 2007 : 26-27.

Philipp M, Bocher J, Siegismund HR \& Nielsen LR, 2006. Structure of a plant-pollinator network on a pahoehoe lava desert of the Galapagos Islands. Ecography 29(4) : 531-540.

Praz C, Müller A, Danforth B, Griswold TL, Widmer A \& Dorn S, 2008. Phylogeny and biogeography of bees of the tribe Osmiini (Hymenoptera: Megachilidae). Molecular Phylogenetics and Evolution 49(1) : 185-197.

Raw A, 2004. Ambivalence over Megachile. Dans BM Freitas \& JOP Fereira, Solitary bees - Conservation, rearing and management for pollination. Federal University of Ceara, Brazil, pp.175-184.

Schwarz MP, Fuller S, Tierney SM \& Cooper SJB, 2006. Molecular phylogenetics of the exoneurine allodapine bees reveal an ancient and puzzling dispersal from Africa to Australia. Systematic Biology 55: 31-45.

Tsuneki K, 1970. Bionomics of some species of Megachile, Dasypoda, Colletes and Bombus. Etizenia 48 : 1-20.

Wu Y, 2005. Fauna Sinica: Insecta Volume 44: Hymenoptera Megachilidae. Beijing [En Chinois, accompagné d'un résumé en Anglais].

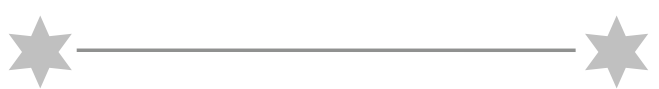

Mangum WA \& Brooks RW, 1997. First records of Megachile (Callomegachile) sculpturalis SMITH (Hymenoptera: Megachilidae) in 